\title{
Conocimientos, Actitudes y Prácticas sobre la Sexualidad en una Población Adolescente Escolar
}

\author{
An adolescent student population's knowledge, attitudes and \\ practice regarding sexuality
}

Juan C. González

Recibido 29 Mayo 2008/Enviado para Modificación 29 Noviembre 2008/Aceptado 10 Enero 2009

\section{RESUMEN}

Objetivo Estimar la relación entre conocimientos, actitudes y prácticas (comportamientos) de riesgo en la sexualidad juvenil y la estructura y percepción de funcionalidad familiar.

Metodología Se realizó un estudio observacional de corte trasversal entre 13699 adolescentes (10 a 19 años de edad) de 12 colegios públicos de la localidad de Suba. Se aplicó una encuesta autodiligenciada donde se interrogó por conocimientos, actitudes y comportamientos sexuales y la estructura y percepción del funcionamiento familiar.

Resultados Edad media de los adolescentes 13,5 años. Prevalencia de relaciones sexuales de $17 \%$. El $8 \%$ de los hogares presentan disfunción severa y $39 \%$ son nucleares incompletos. Las mujeres tienen menos relaciones sexuales que los hombres (OR 0.53; IC $95 \%$; 0.47-0.59). Se presenta más relaciones sexuales cuando los adolescentes perciben de manera disfuncional a su familia (OR 2.06; IC $95 \% ; 1.72-2.48$ ).

Conclusiones Es más importante la percepción de función familiar que la estructura como factor de riesgo en la sexualidad de los jóvenes.

Palabras Clave: Adolescentes, sexualidad, conocimientos, actitudes y prácticas, relaciones familiares, colegios (fuente: DeCS, BIREME).

\section{ABSTRACT}

Objective Assessing the relationship between knowledge, attitudes and practice regarding high-risk sexual behaviour in adolescent sexuality as well as the structure and perception of family functionality.

Methods Cross-sectional research was carried out on a population of 13,699 adolescents (ages ranging from 10 to 19) attending 12 public schools from the conurbation of Suba. A self-questionnaire was administered, focusing on sexual behaviour, knowledge, attitudes and the structure and perception of family dynamics. Results Average age was 13.5 . Sexual activity prevalence was $17 \% .8 \%$ of households suffered from severe dysfunction. $39 \%$ of households were incomplete nuclear ones. Females reported less sexual activity than males (0.53 OR; 0.47-0.59 95\% Cl). Sexual 
activity was more common when adolescents perceived family dysfunction (2.06 OR; 1.72-2.48 95\%Cl).

Conclusions Perception of family function was more important than structure as a risk factor in youngsters' sexuality.

Key Words: Adolescent, sexuality, health knowledge, attitude, practice, family relationships, school (source: $M e S H, N L M$ ).

$\mathrm{L}$

a sociedad colombiana está viviendo un problema en torno a sus jóvenes: se trata del aumento de los embarazos no deseados (tasa de 90 por mil en el 2005) (1) y de la incidencia de enfermedades de transmisión sexual (2) situación que confronta la efectividad de los programas de educación sexual (3) y que se comparte en diversos países occidentales. El asunto es que no parece estar sucediendo lo que se esperaba, es decir una disminución de estos dos eventos gracias a la adquisición de conocimientos. Si bien la mayoría de los jóvenes han recibido información acerca del SIDA o de métodos anticonceptivos antes de iniciar su vida sexual (4), el 80 \% (5) de jóvenes mantienen su primera relación sexual sin protección (y no es planeada). En los colegios en Bogotá, la tendencia educativa se dirige con mayor énfasis (5) hacia promoción de valores (respeto y dignidad) que hacia aspectos biologistas (ciclo menstrual o métodos anticonceptivos). Existe, según el estudio de Mejía (5), opiniones divididas en los padres acerca de la efectividad de los conocimientos de los cursos de educación sexual entregados en los colegios y de hecho algunos consideran que tal información promueve comportamientos de riesgo ("abren" la curiosidad). De la misma manera, la percepción de los profesores es que los padres no promueven diálogos sobre la sexualidad con los jóvenes. Lo cierto es que aunque son los padres y los profesores las principales fuentes de información al respecto, la forma como mayormente se "socializa" (y se aplica) el conocimiento es a través de los pares (5).

Resulta evidente que hace falta indagar más acerca de los diversos componentes que intervienen en los conocimientos y prácticas de los jóvenes. El papel de la familia, sin duda importante en la adquisición de habilidades de los adolescentes y por ende en el rol de protección o de riesgo (6), requiere ser identificado a través de "medidas" que permitan evaluar su participación. Por ello resulta importante analizar nuevas relaciones, lo cual constituye el objetivo esencial de esta investigación, la que pretende estimar las relaciones entre hogares nucleares completos e incompletos, hogares reconstituidos (estructura familiar) y la percepción del adolescente en cuanto a la funcionalidad (percepción de apoyo, afecto, participación, convivencia) con los conocimientos, actitudes y prácticas 
(comportamientos) de sexualidad de la población escolar pública de la localidad de Suba mostrando de esta manera el rol familiar en la sexualidad juvenil.

\section{MATERIAL Y METODOS}

Tipo de estudio: Se trata de un estudio observacional, de corte transversal en adolescentes escolarizados ubicadas de la localidad de Suba (ciudad de Bogotá).

Población de estudio: Fueron seleccionados 13699 jóvenes entre los 10 y los 19 años vinculados a 12 colegios públicos (de un total de 25 probables) de la localidad de Suba, pertenecientes a familias de estratos 1 y 2 en su mayoría.

Criterios de inclusión: Esta investigación forma parte del proyecto PIPSA (Programa Integral de Promoción de Salud del Adolescente) que se viene adelantando desde el 2005 en la localidad de Suba por parte de la facultad de medicina de la Fundación Universitaria Juan N Corpas. Para tal efecto se invitó a participar a los 25 colegios públicos de la localidad a través de las orientadoras, vinculándose 12 colegios. Aceptado el estudio por las directivas del colegio se acudió a los salones de secundaria y se explicó la investigación, así como la libertad para participar en ella y el manejo confidencial del cuestionario. El criterio de inclusión fue el pertenecer al colegio, estar en alguno de los cursos y querer participar en el estudio.

Variables de estudio: Como independientes fueron seleccionadas variables demográficas de edad, género y estratos socioeconómicos (escala de 1 a 6 , expresando los más bajos un mayor nivel de pobreza) y variables referentes a la estructura familiar midiendo la presencia de familias nucleares (padre, madre e hijos), completas o incompletas (ausencia de uno de los padres), familias reconstituidas (padrastros o madrastras) y función familiar usando el test de APGAR familiar que mide a través de cinco preguntas la percepción del joven en la ayuda que siente de la familia para resolver problemas, para participar en decisiones, para sentir afecto, para compartir (7) pudiendo en cada pregunta contestar casi siempre ( 2 puntos), a veces ( 1 punto) y casi nunca ( 0 puntos) y donde se define como función familiar normal un resultado (al sumar las cinco respuestas) entre 8 y 10 disfunción moderada de 4 a 7 puntos y disfunción severa de 0 a 3 puntos. Como variables dependientes se elaboró un cuestionario de 56 preguntas acerca de conocimientos, prácticas, situaciones y actitudes de riesgo de la población adolescente. Para el presente trabajo se seleccionaron las preguntas acerca de conocimientos, actitudes y prácticas de sexualidad: haber recibido información sobre planificación familiar, edad cuando la recibió, fuente 
de la información (8), entendimiento de los métodos de anticoncepción (9), reconoce ciclo menstrual a través de un ejemplo (10) y edades de riesgo en el embarazo (8), protección del condón contra SIDA (4), edad de la madre en primer embarazo (11), deseo de tener un hijo en el próximo año (6), percepción de aceptación de los padres acerca de uso de método de planificación (8); de prácticas a través de las variables: haber tenido relaciones sexuales (5), edad de inicio (12), haber planificado en primera relación (5), método empleado en primera relación (12), relaciones con más de una pareja en el último año (13).

Prueba piloto: En diciembre del 2005 y enero del 2006 se hizo una prueba piloto de instrumentos (encuesta y talleres) con 30 jóvenes de uno de los colegios públicos de la localidad. Se aplicó la encuesta, se identificaron las áreas del cuestionario donde existía alguna confusión, se corrigieron y se obtuvo el formulario definitivo.

Recolección de la información: Las encuestas se llevaron a cabo entre los meses de febrero y junio del 2006. Cada encuesta autodiligenciada tomó en promedio 20 minutos. Para llegar a los colegios se capacitó a un grupo de seis agentes comunitarios (personal proveniente de la comunidad). Al llegar al salón donde estaban los jóvenes, los agentes les pedían a los maestros que salieran y se entregaban los formularios. Se ofreció un programa de consulta para los jóvenes que al llenar la encuesta percibiesen que deseaban ser asistidos médicamente. Cada colegio participante recibió el informe diagnóstico de riesgos.

Manejo de la información: una vez se recibió la encuesta, se tabuló en el programa EXCEL construyendo una base de datos para cada colegio. Se seleccionaron las preguntas acerca de comportamientos de riesgo, las que fueron sometidas al proceso del análisis con EPI INFO. Se obtuvieron las frecuencias de los mencionados comportamientos de manera consolidada para cada colegio. Para cada categoría de las variables dependientes se realizó el análisis multivariado, regresión logística independiente donde se incluyeron todas aquellas variables que en el análisis univariado tuvieron un valor de p menor de 0,02.

\section{RESULTADOS}

La edad media de los jóvenes escolares es de 13,5 años (DS de 1,97), con una moda de 14 años y una mediana de 13 años. 
La mayoría de la población es de estratos 1 y 2 (Tabla 1). La mayoría de las familias de los adolescentes están constituidas por padre, madre e hijos (nucleares). El $39 \%$ son hogares donde falta uno de los padres (estructura). Los hogares reconstituidos habitualmente son por la vinculación de un padrastro (8 veces por cada madrastra). El $15 \%$ de los jóvenes son hijos únicos. Cuando los hogares se convierten en extensos, los abuelos son en el $75 \%$ los miembros de familia vinculados, seguidos de tíos y primos en el $25 \%$ restante. El $38 \%$ de los encuestados son los hijos mayores del hogar (ciclo familiar con hijo adolescente). Por cada joven que percibe a su familia con disfunción severa existe 8,6 que la perciben con buena función.

Tabla 1. Características sociodemográficas y estructura y función familiar de la población adolescente escolar pública de la localidad de Suba en el año 2006

\begin{tabular}{lrr}
\hline Caracteristicas & $\mathbf{N}^{\circ}$ & $\%$ \\
\hline Sociodemográficas & & \\
Edad & 4481 & 33 \\
De 10 a 12 años & 8029 & 59 \\
De 13 a 16 años & 1009 & 8 \\
De 17 a 19 años & 10460 & 79 \\
Estratos & 2654 & 20 \\
$\quad$ Estratos 1 y 2 & 76 & 1 \\
Estratos 3 & & \\
Estratos 4 o más & 6944 & 51 \\
Género & & \\
Femenino & 7619 & 59 \\
Estructura Familiar & 5386 & 41 \\
Familia Nuclear: & 2854 & 21 \\
$\quad$ Completa & 1236 & 9 \\
Incompleta & 1065 & 8 \\
Familia Extensa & 171 & 1 \\
Familia Reconstituida & & \\
Con padrastro & & \\
Con madrastra & & \\
Función Familiar & 3590 & 68 \\
Buena función & 998 & 24 \\
Disfunción moderada & & 8 \\
Disfunción severa & & \\
\hline
\end{tabular}

La edad media cuando reciben información acerca de planificación son los 12 años (Tabla 2). El colegio y los padres son los principales responsables de ofrecer la información sobre sexualidad (Tabla 2). Un 71 \% de los adolescentes no reconocen con certeza protección derivada del condón. Los hombres inician su vida sexual a una edad media de 13 años, las mujeres a los 14 años y uno de cada tres de los que inician la vida sexual, no planificaron en la primera relación. El condón es el principal método de planificación y protección empleado. 
Tabla 2. Frecuencias de conocimientos, actitudes y prácticas relacionadas con la sexualidad de la población adolescente escolar pública de la localidad de Suba en el año 2006

\begin{tabular}{|c|c|c|}
\hline Variable & $\mathrm{N}^{\circ}$ & $\%$ \\
\hline \multicolumn{3}{|l|}{ Conocimientos y actitudes } \\
\hline \multicolumn{3}{|l|}{ Ha recibido información sobre cómo planificar: } \\
\hline Sí y la entendió & 4768 & 36 \\
\hline Si, pero tiene dudas & 3295 & 25 \\
\hline No ha recibido & 5051 & 38 \\
\hline \multicolumn{3}{|l|}{ Quién le enseñó sobre planificación } \\
\hline Colegio & 5236 & 38 \\
\hline Padres & 4179 & 31 \\
\hline Por su cuenta & 969 & 7 \\
\hline Servicio de salud & 849 & 6 \\
\hline Amigos & 793 & 6 \\
\hline No entiende los métodos de planificación & 4621 & 52 \\
\hline Reconoce edades de embarazo de alto riesgo & 2719 & 21 \\
\hline Entiende el ciclo menstrual (a través de preguntas) & 1711 & 13 \\
\hline \multicolumn{3}{|l|}{ Cree que el condón protege contra el SIDA } \\
\hline Sí lo cree & 3909 & 30 \\
\hline Lo cree pero no esta seguro & 4832 & 37 \\
\hline No lo cree & 2224 & 17 \\
\hline No sabe & 2221 & 17 \\
\hline Madre embarazada antes de 18 años & 1861 & 14 \\
\hline Desea tener un hijo en los próximos 12 meses & 264 & 2 \\
\hline $\begin{array}{l}\text { Cree que los padres lo apoyarían si decidiera } \\
\text { planificar }\end{array}$ & 4586 & 34 \\
\hline No cree que lo apoyen & 1814 & 14 \\
\hline No sabe qué pensarían & 6900 & 52 \\
\hline \multicolumn{3}{|l|}{ Prácticas (Comportamientos) } \\
\hline Ha tenido relaciones sexuales & 2224 & 17 \\
\hline No planificó en la primera relación sexual & 971 & $33^{a}$ \\
\hline \multicolumn{3}{|l|}{ Método que empleó en la primera relación sexual } \\
\hline Condón & 1079 & $71^{b}$ \\
\hline Coitus interruptus & 329 & 22 \\
\hline Pastilla & 28 & 2 \\
\hline Inyección & 75 & 5 \\
\hline Otros & 36 & 2 \\
\hline $\begin{array}{l}\text { Relaciones sexuales con más de una persona en el } \\
\text { último año }\end{array}$ & 550 & 4 \\
\hline
\end{tabular}

${ }^{\mathrm{a}} \mathrm{De}$ los que han tenido relaciones sexuales; ${ }^{\mathrm{b}} \mathrm{Del}$ total que planificaron

Las mujeres han sido mejor informadas sobre cómo planificar (Tabla 3) que los hombres, creen menos en la protección del condón y perciben un menor apoyo de sus padres respecto a la decisión de planificar. Han tenido menos relaciones sexuales que los hombres, así como tienen menos parejas sexuales en el último año. 
Tabla 3. Razones de riesgo. Análisis de regresión logística incondicional de conocimientos, actitudes y prácticas y género en adolescentes escolar pública de la localidad de Suba. 2006

\begin{tabular}{|c|c|c|c|c|c|c|c|c|}
\hline \multirow{2}{*}{ VARIABLE } & \multicolumn{2}{|c|}{ SI } & \multicolumn{2}{|c|}{ No } & \multirow{2}{*}{ OR } & \multicolumn{2}{|c|}{ IC $95 \%$} & \multirow{2}{*}{$\begin{array}{c}\text { Valor } \\
P\end{array}$} \\
\hline & $\mathrm{N}^{\circ}$ & $\%$ & $\mathrm{~N}^{\circ}$ & $\%$ & & $\mathrm{LI}$ & LS & \\
\hline \multicolumn{9}{|l|}{ Conocimientos y actitudes } \\
\hline \multicolumn{9}{|l|}{$\begin{array}{l}\text { Ha recibido Información } \\
\text { sobre cómo planificar }\end{array}$} \\
\hline Femenino & 2437 & 52 & 2284 & 48 & \multirow{3}{*}{1,4} & \multirow{3}{*}{1,2} & \multirow{3}{*}{1,6} & \multirow{3}{*}{0,000} \\
\hline Masculino & 2317 & 46 & 2726 & 54 & & & & \\
\hline Casos incluidos: 7630 & & & & & & & & \\
\hline \multicolumn{9}{|l|}{ Cree que el condón } \\
\hline protege contra el Sida & & & & & \multirow{4}{*}{0,4} & \multirow{4}{*}{0,3} & \multirow{4}{*}{0,4} & \multirow{4}{*}{0,000} \\
\hline Femenino & 1505 & 37 & 2509 & 63 & & & & \\
\hline Masculino & 2383 & 57 & 1829 & 43 & & & & \\
\hline Casos incluidos: 6409 & & & & & & & & \\
\hline \multicolumn{9}{|l|}{ Cree que los padres lo } \\
\hline \multicolumn{9}{|l|}{$\begin{array}{l}\text { apoyarian si decidiera } \\
\text { planificar }\end{array}$} \\
\hline Femenino & 2183 & 69 & 986 & 31 & \multirow{3}{*}{0,7} & \multirow{3}{*}{0,6} & \multirow{3}{*}{0,8} & \multirow{3}{*}{0,000} \\
\hline Masculino & 2382 & 74 & 817 & 26 & & & & \\
\hline \multicolumn{5}{|l|}{ Casos incluidos: 5271} & & & & \\
\hline \multirow{2}{*}{\multicolumn{9}{|c|}{$\begin{array}{l}\text { Comportamientos } \\
\text { Relaciones sexuales }\end{array}$}} \\
\hline & & & & & & & & \\
\hline Femenino & 865 & 13 & 5930 & 87 & \multirow{3}{*}{0,5} & \multirow{3}{*}{0,5} & \multirow{3}{*}{0,6} & \multirow{3}{*}{0,000} \\
\hline Masculino & 1334 & 20 & 5190 & 80 & & & & \\
\hline \multicolumn{5}{|l|}{ Casos incluido s: 10519} & & & & \\
\hline \multicolumn{9}{|l|}{ Más de una pareja } \\
\hline sexual al año & & & & & & & & \\
\hline Femenino & 156 & 17 & 743 & 83 & \multirow{2}{*}{0,5} & 14 & 07 & 000 \\
\hline $\begin{array}{l}\text { Masculino } \\
\text { Casos incluidos :1816 }\end{array}$ & 389 & 28 & 996 & 72 & & 0,4 & 0,1 & 0,000 \\
\hline
\end{tabular}

La Tabla 4 evidencia la ausencia de relaciones significativas de la estructura familiar (ni en los hogares nucleares completos e incompletos ni en los reconstituidos) con los conocimientos, actitudes y prácticas indagados. En relación con la función familiar se encontró que la percepción disfuncional de la familia del adolescente se relaciona con querer tener un hijo ese año, con la percepción de no apoyo de padres a la decisión de planificar y con más relaciones sexuales.

\section{DISCUSIÓN}

El principal sesgo que enfrenta esta investigación es que los jóvenes no contesten con la verdad. Su diseño subsanó esto a través del cumplimiento del anonimato de cada formulario, de la insistencia en la garantía de la voluntariedad, de la exclusión de profesores en la vigilancia al momento de hacer la encuesta, del diseño de la encuesta el cual permitió la interacción de los jóvenes incentivando 
el deseo de contestarla (obtenían apreciaciones del riesgo a que están expuestos). A pesar del tamaño de la población en estudio no es una muestra representativa.

Tabla 4. Razones de riesgo. Análisis de Regresión logística incondicional de Conocimientos, Actitudes y Prácticas y la familia (estructura y función) en adolescentes escolares de la localidad de Suba. 2006

\begin{tabular}{|c|c|c|c|c|c|c|c|c|}
\hline \multirow{2}{*}{ Variable } & \multicolumn{2}{|l|}{ sí } & \multicolumn{2}{|c|}{ NO } & \multirow{2}{*}{ OR } & \multicolumn{2}{|c|}{ IC $95 \%$} & \multirow{2}{*}{$\begin{array}{c}\text { Valor } \\
P\end{array}$} \\
\hline & $\mathrm{N}^{\circ}$ & $\%$ & $\mathrm{~N}^{\circ}$ & $\%$ & & LI & LS & \\
\hline \multicolumn{9}{|l|}{$\begin{array}{l}\text { Conocimientos y } \\
\text { actitudes }\end{array}$} \\
\hline \multicolumn{9}{|l|}{$\begin{array}{l}\text { Quiere tener un hijo } \\
\text { este año }\end{array}$} \\
\hline $\begin{array}{l}\text { Disfunción severa } \\
\text { Funcional } \\
\text { Casos incluidos: } 3690\end{array}$ & $\begin{array}{r}41 \\
132\end{array}$ & $\begin{array}{r}10 \\
5\end{array}$ & $\begin{array}{r}369 \\
2689\end{array}$ & $\begin{array}{l}90 \\
95\end{array}$ & 2.2 & 1,5 & 3,4 & 0,000 \\
\hline \multicolumn{9}{|l|}{$\begin{array}{l}\text { Cree que padres } \\
\text { apoyarían planificación }\end{array}$} \\
\hline $\begin{array}{l}\text { Disfunción severa } \\
\text { Disfunción moderada }\end{array}$ & $\begin{array}{r}345 \\
1120\end{array}$ & $\begin{array}{l}66 \\
70\end{array}$ & $\begin{array}{l}182 \\
475\end{array}$ & $\begin{array}{l}35 \\
30\end{array}$ & 0,7 & 0,6 & 0,8 & 0,000 \\
\hline $\begin{array}{l}\text { Disfunción severa } \\
\text { Funcional } \\
\text { Casos incluidos: } 5271\end{array}$ & $\begin{array}{r}345 \\
2889\end{array}$ & $\begin{array}{l}66 \\
73\end{array}$ & $\begin{array}{r}182 \\
1046\end{array}$ & $\begin{array}{l}34 \\
27\end{array}$ & 0,6 & 0,5 & 0,7 & 0,000 \\
\hline \multicolumn{9}{|l|}{ Prácticas } \\
\hline $\begin{array}{l}\text { Disfunción severa } \\
\text { Disfunción moderada }\end{array}$ & $\begin{array}{l}295 \\
612\end{array}$ & $\begin{array}{l}30 \\
21\end{array}$ & $\begin{array}{r}685 \\
2363\end{array}$ & $\begin{array}{l}70 \\
79\end{array}$ & 1,2 & 1 & 1,3 & 0,002 \\
\hline $\begin{array}{l}\text { Disfunción severa } \\
\text { Funcional } \\
\text { Casos incluidos: } 10519\end{array}$ & $\begin{array}{r}295 \\
1188\end{array}$ & $\begin{array}{l}30 \\
14\end{array}$ & $\begin{array}{r}685 \\
7262\end{array}$ & $\begin{array}{l}70 \\
86\end{array}$ & 2 & 1,7 & 2,5 & 0,000 \\
\hline
\end{tabular}

Al comparar las variables de la familia (Tabla 1) con el estudio efectuado en esta misma localidad y en los mismos estratos en el 2002 (15) se observa un descenso de los hogares nucleares (68\% en el 2002) e incremento de los hogares nucleares incompletos ( $28 \%$ en el 2002) e incremento de hogares extensos $(16 \%$ en el 2002). Estas diferencias pueden reflejar una tendencia a la separación en esta población. El ciclo familiar en este estudio es similar al encontrado en el estudio del 2002 (15), cuando se encontró la misma proporción de hogares cuyo hijo mayor es el adolescente (38 \% en ambos momentos). En cuanto a la percepción de la funcionalidad, esta investigación refleja que la crisis normativa (16) que se presenta en la adolescencia (enfrentamiento con padres y hermanos) por parte del joven se refleja con un porcentaje de disfunción severa del $8 \%$ (IC $95 \%$ de 7.4-8.4) y de buena función de un $68 \%$ (IC $95 \%$ 67,3-68,9) situación que puede servir de referente de la función familiar para los adolescentes de 13,5 años (edad media) de los colegios públicos estudiados. 
Entre los 10 y los 19 años los jóvenes reciben, interpretan y vinculan la información acerca de su sexualidad y, además con la influencia generada por la sociedad, la familia y los pares, desarrollarán su vida sexual, asumiendo los riesgos y protecciones correspondientes (9). Esta investigación incluye jóvenes de 10 a 12 años (Tabla 1) siendo entre los 12 y los 13 años el momento donde se inician los programas más intensivos de educación sexual a nivel escolar. Ello explica que existan cerca de un $38 \%$ de adolescentes que no han recibido información sobre planificación. En Colombia al igual que otras partes de Latinoamérica (17) los jóvenes (de 13 a 19 años) reciben información acerca de métodos de anticoncepción a nivel del colegio, siendo reforzado por la familia, pero y a pesar de ello, el $52 \%$ no entiende los métodos de anticoncepción (indagado a través de preguntas específicas) así como no identifican la importancia del condón como factor protector de ETS (17 \% no cree y $37 \%$ no está seguro) cifra similar a la encontrada por Mosquera en el 2003 (8), en donde el 41 $\%$ no creen que proteja y $11 \%$ expresan tener dudas. Se plantea que existe un problema o bien con la forma o con el momento en que se ofrece esta información: lo que se usa para enseñar y cuándo se da, no llega a cerca de la mitad de los jóvenes (Tabla 2). Reflejan estas cifras la desinformación existente sobre el condón a nivel de profesores y padres. Una explicación del por qué esta situación la da Motta (9) quien revisó que la información sobre planificación se da alrededor de los 13 años en Brasil, edad en donde un porcentaje de jóvenes no han iniciado la vida sexual y por ende la información no es percibida cómo útil (aún), no recibiéndola de nuevo con la misma insistencia más adelante (cuando se inician las relaciones sexuales). La edad media cuando se da la información en esta investigación fueron los 12 años un año antes de la media de inicio de relaciones sexuales lo que puede determinar que no les parezca "útil" en ese momento. Corrobora esta hipótesis el hecho de que entre más años del joven, mejor asimilan el conocimiento (17). De todas maneras, la información "sola" no resuelve el problema de la protección. Trajman (4) encontró que un 94 \% de los jóvenes escolares en Brasil saben de la protección contra el SIDA que ofrece el condón, sin embargo, solo el 34 \% de los jóvenes lo usan siempre, situación similar a la encontrada por Burbano (18) quien en Bogotá, muestra cómo en un grupo de mujeres con comportamientos de alto de riesgo y con conocimientos "sólidos" sobre el condón, un 29 \%, no lo usó en última relación. Florez (19) revisó que existe en algunos jóvenes una percepción negativa hacia el uso del condón ("no se siente igual" y "no está bien usarlo con la pareja").

Ortiz (20) detectó que en un $74 \%$ de las adolescentes embarazadas, la madre de ellas tuvo su primer hijo en la adolescencia, por ello el $14 \%$ encontrado (Tabla 2) es un factor de riesgo confirmado por Florez (21) en su estudio de 
gestantes adolescentes de Cali y Bogotá. Así mismo, encontró que el 59 \% de las gestantes deseaban tener un hijo en ese año situación que hace ver como población de riesgo de gestación a ese $2 \%$ encontrado en este estudio (Tabla 2). El apoyo de los padres a la planificación es un factor protector (8) presente casi siempre en los hombres (Tabla 3) y menos en las mujeres. El $17 \%$ de los jóvenes ya iniciaron su vida sexual siendo los 13 años la media, situación que se confirma con el estudio de Profamilia (1) del 2005, situación que confirma el adelanto del inicio de la vida sexual, comparado en las mujeres de 20 a 45 años la edad, cuyo inicio fue a los 18 años. Factores culturales sin duda influyen y en general la tendencia en el mundo occidental es a un inicio más temprano (22) en donde, por ejemplo, la edad de 13 años presenta unas prevalencias de inicio de $20 \%$ en estudiantes japoneses, $34 \%$ en nigerianos, $43 \%$ en ecuatorianos, $45 \%$ en suizos $37 \%$ en estudiantes estadounidenses ( $41 \%$ en afro americanos e hispanos), 11 $\%$ en adolescentes chilenos y $18,4 \%$ en estudiantes mexicanos. Probablemente los medios de comunicación tengan que ver con ello y, Florez (21) demuestra en su estudio cómo los jóvenes consideran por completo normal esta situación. Así mismo en su investigación describe cifras similares a la encontrada en la pregunta de ausencia de protección en la primera relación sexual (Tabla 2). Como se observa, los datos obtenidos no muestran que se haya logrado modificar este comportamiento. ¿Será que no se hace lo suficiente? $\mathrm{O}$, ¿que lo que se hace no tiene el impacto necesario? Este aspecto amerita ser estudiado para ser intervenido, máxime si como dice Florez (21) algunos jóvenes creen que por ser la primera relación "nada" les va a pasar, con lo cual se promueve la relación sin protección y la mayoría inician procesos de planificación un año después de iniciar su vida sexual. La anterior se corrobora en el estudio de Mejía (5) quien encontró que 1 de cada 4 jóvenes quedan embarazadas en la primera relación. En este estudio (Tabla 2) un $4 \%$ de los jóvenes tuvieron relaciones sexuales con más de una pareja en el ultimo año dato importante como referente. En Cuba según el estudio de Cándido (23) este porcentaje es 4 veces mayor, siendo la explicación mayor edad media de la población.

El análisis de las relaciones derivadas de la diferencia de sexo (Tabla 3), confrontan de manera interesante el mundo biológico con el mundo "social". Ambos, hombre y mujer, sienten el influjo de las hormonas, pero son los hombres los que asumen mayores comportamientos de riesgo. Entre las posibles explicaciones se ofrecen estas dos: o que los hombres tiene más "impulso biológico" o que las mujeres están más inhibidas socialmente en torno a sus comportamientos sexuales (lo que se convierte en un factor protector). Relaciones similares son las halladas en los comportamientos violentos (24). Si bien socialmente se permite más la violencia en los hombres que en las mujeres, el hallazgo combinado 
permite plantear que estos comportamientos estén bajo el "influjo" hormonal de la testosterona.

En torno a la pregunta de investigación se confirma que es la forma como funciona y no la estructura el factor clave de protección y/o riesgo de los adolescentes en torno a su familia (Tabla 4). Urrea (25) sostiene que en los hogares nucleares completos los adolescentes inician las relaciones sexuales más tarde (comparados los incompletos vs los completos), situación no encontrada en este trabajo (OR de 1,07 con IC $95 \%$ de 0.9-1.2). Florez (21) sostiene lo mismo, pero en los jóvenes de Cali y no así en los de Bogotá, diferencia interesante que puede mostrar cómo la familia es influenciada por culturas regionales. Florez sostiene que es la funcionalidad el factor protector esencial en torno a los comportamientos sexuales de riesgo y repite el inmenso riesgo de la agresión física en el hogar hacia jóvenes para "permitirse" quedar embarazadas (en esta investigación el 2 $\%$ de los jóvenes afirmaron ser golpeados regularmente por su padres). Una explicación puede ser que la joven considere el embarazo como una salida de su actual hogar. Lo cierto es que este trabajo permite ver cómo la disfunción familiar también afecta la comunicación (Tabla 4) reflejada en no creer que los padres apoyarían la decisión de planificar y, por supuesto, cómo incrementan los riesgos de los comportamientos.

Esta investigación confirma la tendencia del inicio de la vida sexual más temprano. Que la información recibida no retrasa el inicio de la vida sexual. Que la información se da pero existen problemas en el proceso pues los jóvenes no tienen los conocimientos esperados. Que no se está usando el condón como y en las circunstancias que se esperaba. Respecto a la pregunta planteada, que prima la función sobre la estructura, siendo la disfunción familiar un factor de riesgo moderado de comportamientos en torno a la sexualidad

Agradecimientos. A la rectoría de la Fundación Universitaria Juan N Corpas que junto con la Fundación Salud Familia y Comunidad financiaron esta investigación. A las orientadoras de los colegios públicos de la localidad de Suba que han insistido en vincular a la comunidad en pos de soluciones para los adolescentes de sus colegios. 


\section{REFERENCIAS}

1. Profamilia. Salud Sexual y Reproductiva en Colombia: Encuesta Nacional de Demografía y Salud; 2005.

2. Ministerio de Protección Social de Colombia. Norma técnica para la detección temprana de las alteraciones del desarrollo del joven de 10 a 29 años; 2000.

3. Senderowitz J. A review of program approaches to adolescent reproductive health. Preparado por: U.S. Agency for International Development Population Technical Assistance Project Bureau for Global Programs; 2000.

4. Trajman A, Belo MT, Teixeira EG, Dantas VD, Salomão FM, LedoAJ, et al. Knowledge about STD/ AIDS and sexual behavior among high school students in Rio de Janeiro, Brazil. Cad. Saúde Pública 2003; 19(1).

5. Mejía IE. Dinámicas ritmos y significados de la sexualidad juvenil. Programa La Casa CESO. Universidad de los Andes; 2000.

6. Krauskoph D. Conductas de riesgo en fase juvenil. Documento OPS 2002. [Internet]. Disponible en: http://www.ilo.org/public/spanish/region/ampro/cinterfor/temas/youth/doc/not/libro37/ libro37.pdf. Consultado Marzo del 2006.

7. Smilkstein G. The Family APGAR: A proposal for a family function test and its use by physicians. J Fam Pract 1978; 17: 1151.

8. Mosquera J, Mateus JC. Conocimientos, actitudes y prácticas sobre métodos de planificación familiar, VIH y sida y el uso de los medios de comunicación en jóvenes. Revista Colombia médica 2003; 34(4).

9. Motta LB, Costa-Paivab L, Osisc MJ, Sousac MH, Netob P, ValdirT. Knowledge of contraceptive methods among adolescent students. Rev Saudé Publica 2006; 40 (1).

10. Martínez O, Franco A, Franco A, Silva J, Velásquez G. Embarazo en la adolescencia y conocimientos sobre sexualidad. Revista Cubana de Medicina Integral 2003; 19(6).

11. Ortiz R, Anaya NI, Sepúlveda C, Torres SJ, Camacho PA. Caracterización de las adolescentes gestantes de Bucaramanga, Colombia. Un estudio transversal. Revista MedUnab 2005; $8(2)$.

12. González C, Rojas R, Hernández MI, Olaiz G. Perfil del comportamiento sexual en adolescentes mexicanos de 12 a 19 años de edad. resultados de la ENSA 2000. Revista Salud Pública de México 2005; 47(3).

13. Soto O, Franco A, Franco A, Silva J, Velásquez GA, Zúñiga. Embarazo en la adolescencia y conocimientos sobre sexualidad Revista cubana de medicina integral. 2003; 19 (6).

14. Mejía Soto $G$ Propuesta de una estrategia que permita detectar abuso sexual en adolescentes. informe preliminar Acta Pediatr Mex 2005; 26(2):90-95.

15. González JC, Álvarez AC. Diagnóstico biopsicosocial de 437 familias de la UPZ 71. Investigación elaborada para BAS (Bogotana de Aguas Sanitarias) en el año 2002. Disponible en http:/ /www.juanncorpas.edu.co/index.php?id=1295 Consultada Marzo 2006.

16. Mejía D. Salud familiar para América Latina. Edición ASCOFAME; Colombia 1991.

17. Moccia A, Medina R. Qué saben las adolescentes acerca de los métodos anticonceptivos y cómo los usan. Rev Med Urug 2006; 22:185-190.

18. Burbano MJ, de Pool I, Hadrigan S, Jackson J Jr, Angarita I, Then EP, et al. HIV knowledge and risk behaviors among women in law enforcement in Bogotá, Colombia: potential role as community educators. Urban Health 2005; 82:43-57.

19. Florez CE, Soto V, Vargas E, Henao J, González C. Fecundidad adolescente en Colombia: incidencia, tendencias y determinantes. Un enfoque de historia de vida. Bogotá: Centro de Estudios de Desarrollo Económico/Departamento de Psicología, Universidad de los Andes; 2004

20. Ortiz R, Anaya NI, Sepúlveda C, Torres SJ, Camacho PA. Caracterización de las adolescentes gestantes de Bucaramanga, Colombia. Un estudio transversal. Revista MED UNAB. 2005; 8(2). 
21. Florez $\mathrm{CE}$. Socioeconomic and contextual determinants of reproductive activity among adolescent women in Colombia. Rev Panam Salud Publica 2005; 8(6).

22. Ceballos GA, Campo A. Relaciones sexuales en adolescentes escolarizados de Santa Marta, Colombia: una encuesta transversal. Colomb. Med 2007; 38(3).

23. Cándido E, Méndez B, Guerra JM, Rodríguez R. Conducta de riesgo en infecciones de transmisión sexual y embarazo. Rev Cubana Med Gen Integr 2003; 19(1).

24. Brook DW, Brook JS, Rosen Z, De la Rosa M, Montoya ID, Whitman M. Early risk factors for violence in Colombian adolescents. Revista American Journal Psychiatry. 2003; 160: 1470-1478.

25. Urrea F, Congolino ML, Herrera HD, Reyes JI, Botero W. Comportamientos sexuales e incidencia de los programas de salud sexual y reproductiva en estudiantes de secundaria de sectores populares y de la universidad pública en la ciudad de Cali, Colombia. Cad. Saúde Pública 2006; 22(1). 